\title{
Inteligencia emocional durante la adolescencia: su relación con la participación en actividades extracurriculares
}

\author{
Alejandra Daniela Caleroํㅡ, Juan Pablo Barreyro² e Irene Injoque-Ricle ${ }^{3}$
}

\begin{abstract}
RESUMEN
La inteligencia emocional percibida se define con una serie de habilidades que permiten manejar y servirse de la información emocional. Está compuesta por la atención a las emociones, la claridad con la que se perciben los sentimientos y la capacidad de reparación de los estados emocionales negativos. La participación en actividades extracurriculares durante la adolescencia se está considerando un recurso para el desarrollo de habilidades y competencias. Objetivo: Estudiar diferencias en la inteligencia emocional percibida según la participación en actividades extracurriculares. Muestra: 399 adolescentes, con una edad media de 15.14 años $(D E=1.86)$ alumnos de dos escuelas privadas de educación media de la Ciudad Autónoma de Buenos Aires. Materiales: Se administró a los adolescentes la TMMS-21 que evalúa los tres componentes de la inteligencia emocional y un cuestionario sociodemográfico para recabar información sobre las actividades extracurriculares realizadas. Resultados: Se hallaron diferencias en el componente de la inteligencia emocional claridad, siendo mayor para aquellos que participan en actividades extracurriculares. También se halló que los adolescentes que participaban en actividades deportivas reportaron mayores niveles de claridad que los que participan en actividades artísticas. Conclusión: La participación en actividades extracurriculares parece brindar a los adolescentes herramientas para desglosar, identificar y catalogar las emociones con más facilidad. Además, la diferencia hallada entre las actividades deportivas y artísticas se puede deber a que el mayor nivel de introspección motivado desde las actividades artísticas puede aumentar el nivel de autocrítica con respecto a la propia capacidad de claridad a la hora de percibir las emociones, reflejado en el reporte de un menor nivel de percepción de capacidad para etiquetar los estados emocionales.
\end{abstract}

Palabras clave: inteligencia emocional, actividades extracurriculares, adolescencia, habilidades emocionales.

\section{Emotional intelligence during adolescence: its relation to participation in extracurricular activities}

\begin{abstract}
Perceived emotional intelligence is a set of skills that allow managing and serving emotional information. It includes attention to emotions, the clarity with which feelings are perceived, and the ability to repair the negative emotional states. Participation in extracurricular activities during adolescence has been considered a resource for the development of skills and competencies. Objective: to study if differences in emotional intelligence perceived according to the participation in extracurricular activities. Sample: 399 adolescents, with a mean age of 15.14 years $(S D=1.86)$ from two private schools in Buenos Aires City. Materials: the TMMS-21, which evaluates the three components of emotional intelligence, and a socio-demographic questionnaire to gather information on extracurricular activities were administered. Results: We found differences in the level of clarity that adolescents reported, being higher for those who participated in
\end{abstract}

1, 2 y 3 Consejo Nacional de Investigaciones Científicas y Técnicas (CONICET), Facultad de Psicología Universidad de Buenos Aires, Argentina; acalero@psi.uba.ar 
extracurricular activities. We also found that adolescents participating in sports reported higher levels of clarity then those involved in artistic activities. Conclusion: Participation in extracurricular activities seems to provide teens with tools to decompose, identify and catalog the emotions more easily. In addition, the difference found between sports and artistic activities may be due to the fact that a higher level of introspection, motivated by the artistic activities, can increase the level of self-criticism regarding one's clarity when it comes to perceiving emotions, which could be reflected in a lower level of perception of the capacity to label different emotional states.

Keywords: emotional intelligence, extracurricular activities, adolescence, emotional skills.

El constructo Inteligencia Emocional fue acuñado por Salovey y Mayer (1990), quienes lo definieron como la capacidad de percibir, entender y controlar las emociones propias y la de los demás, y utilizarlas para guiar los pensamientos y emociones de modo que resulte beneficioso para el individuo y el medio al que pertenece Es la capacidad de entender los significados de los patrones emocionales y de poder razonar y resolver problemas en función de la información que ellos brindan (Mayer, Salovey, \& Caruso, 2000). La inteligencia emocional, desde este modelo, está compuesta por tres aspectos: la percepción sobre la capacidad para atender a los estados emocionales, la percepción sobre la claridad para poder desglosar y etiquetar las emociones y por la percepción sobre la propia capacidad de reparar los estados emocionales negativos (Salovey et al., 1995).

La inteligencia emocional percibida durante la adolescencia ha mostrado ser un constructo importante para el ajuste psicológico (Jiménez \& LópezZafra, 2011; Salguero, Fernández-Berrocal, Ruiz-Aranda, Castillo, \& Palomera, 2011; Salguero, Palomera, \& Fernández-Berrocal, 2012; Williams, FernándezBerrocal, Extremera Pacheco, Ramos Díaz, \& Joiner, 2004). En investigaciones previas se han asociado los niveles de claridad y reparación con bajos niveles de depresión, ansiedad y desajuste en la escuela (Palomera, Salguero, \& Fernández-Berrocal, 2011), con mejores índices de salud mental, niveles más bajos de impulsividad (Fernández-Berrocal, Salovey, Vera, Ramos Díaz, \& Extremera Pacheco, 2002) y con mayores niveles de satisfacción vital (Rey, Extremera Pacheco, \& Pena, 2011).

En el caso de atención a los sentimientos, los resultados parecen indicar que, si el nivel de atención no es acompañado con niveles similares de claridad y recuperación, puede relacionarse con una baja salud mental (Palomera, et al., 2011). Aunque, por otro lado, la atención a las emociones se ha visto asociada a una percepción de mayor adaptación social y conductas prosociales por parte de los docentes de adolescentes (Jiménez \& López-Zafra, 2011).

La adolescencia, junto con la niñez, es un período fundamental para aprender hábitos emocionales, sumados a competencias, recursos psicológicos y conductuales que acompañarán al individuo el resto de su vida (Goleman, 2012; Lozano, Uzquiano, Rioboo, Paz, \& Castro, 2013). Tomando en consideración que las emociones, desde un punto de vista evolutivo, tienen un gran valor adaptativo e informativo con relación a las interacciones con el ambiente (Damasio, 1994; Mayer \& Salovey, 1997; Salovey et al., 1995), los contextos en que los adolescentes participen y las experiencias por las que transiten se vuelven importantes para el desarrollo de distintas competencias, entre las que se encuentran las habilidades emocionales. En la última década se ha observado un mayor reconocimiento de la importancia que desempeñan 
las actividades y los contextos para el desarrollo durante la adolescencia (Benson, 2003; Coleman \& Hendry, 2003; Hansen, Larson, \& Dworkin, 2003). De hecho, las actividades extracurriculares son consideradas un recurso para fomentar el desarrollo positivo del adolescente (Parra Jiménez, Oliva Delgado, \& Antolín Suárez, 2009).

Las actividades extracurriculares, por su parte, se caracterizan por ser autodeterminadas y realizarse por fuera del horario escolar. Las mismas permiten que el adolescente realice un uso del tiempo libre que le facilita un mayor crecimiento, desarrollo (Eccles \& Gootman, 2002; Larson, 2000) e integración social (Gracia \& Herrero, 2006). Son actividades semi estructuradas de participación activa y automotivada que permiten que el adolescente se sienta en control del devenir de la actividades dentro de los limites que la misma impone (Calero, 2016).

Larson y Brown (2007), a partir de una investigación que estudiaba el desarrollo emocional en adolescentes que participaban de una actividad extracurricular, concluyeron que el hecho de que se den situaciones emocionales en un entorno emocionalmente positivo y seguro de forma repetida ayuda a los adolescentes a aprender a identificar las emociones, como así también sus consecuencias y a desarrollar estrategias para controlarlas.

A pesar del interés que en las últimas décadas suscitó el constructo inteligencia emocional y de la importancia que se concede a las actividades en que los adolescentes participan, se carecen de investigaciones que indaguen de forma empírica la relación entre la participación en actividades extracurriculares, y sus características, con la inteligencia emocional. E1 objetivo general del presente trabajo es estudiar diferencias en la inteligencia emocional percibida según la participación en actividades extracurriculares. Los objetivos específicos son comparar la inteligencia emocional en función de si los adolescentes realizan o no actividades extracurriculares; comparar la inteligencia emocional entre los adolescentes que realizan una o más de una actividad; comparar la inteligencia emocional según el tiempo y frecuencia semanal de la actividad extracurricular realizada por los adolescentes; comparar la inteligencia emocional según el tipo de actividad extracurricular; y comparar la inteligencia emocional según los adolescentes posean o no un profesor que guíe la actividad.

\section{MÉTODO}

\section{Tipo de estudio}

El diseño del estudio fue no experimental transversal de tipo descriptivo correlacional de acuerdo a la tipología utilizada en Hernández Sampieri, Fernández-Collado, y Baptista Lucio (2008).

\section{Participantes}

La muestra estuvo compuesta por 399 adolescentes (263 mujeres $65.9 \%-$ y 136 varones $-34.1 \%-)$, con una edad media de 15.14 años $(D E=$ 1.86; Mín = 13 Máx = 19), alumnos de dos escuelas privadas de educación media de la Ciudad Autónoma de Buenos Aires.

La muestra es de tipo no probabilística (o dirigida) por conveniencia (Hernández Sampieri et al., 2008). Es un tipo de muestra no probabilística cuya selección para formar parte del estudio fue determinada por 
conveniencia, es decir, por la disponibilidad de instituciones educativas dispuestas a participar de la investigación.

Participaron aquellos adolescentes que contaron con el consentimiento de sus padres para participar de la investigación. En estos consentimientos se explicaba la naturaleza de la investigación, la participación voluntaria, se garantizaba la confidencialidad de la información y el anonimato de los participantes.

\section{Materiales}

TMMS-21 (Calero, 2013). Es una escala de autoinforme que permite evaluar la inteligencia emocional percibida a través de 21 reactivos que indagan la autopercepción en relación con la capacidad de manejar las emociones y las cualidades estables de las mismas. Posibilita detectar diferencias interindividuales en la manera en que las personas perciben atender las emociones, la claridad con la que lo hacen y la capacidad de reparar los estados emocionales. Evalúa las tres dimensiones de la inteligencia emocional percibida propuesta por Salovey et al. (1995): siete ítems refieren a atención a las emociones (e.g.: "Estoy muy atento/a a mis sentimientos"), siete a claridad emocional (e.g.: "Usualmente sé cuáles son mis sentimientos acerca de una cuestión o situación") y siete a reparación de las emociones (e.g.: "No importa lo mal que me sienta, trato de pensar en cosas agradables"). Al adolescente se le pide que evalúe el grado en el que está de acuerdo con cada uno de los items sobre una escala tipo Likert de 5 puntos desde 1 (totalmente en desacuerdo) hasta 5 (totalmente de acuerdo) a partir de la cual se obtiene una medida de los aspectos reflexivos de la experiencia emocional expuestos anteriormente. Se estimó un coeficiente alfa de Cronbach para cada una de las subescalas, obteniendo un valor de .79 en atención, .86 en claridad y de .86 en reparación. Asimismo, las correlaciones ítem total fueron en todos los casos superiores a .40. El instrumento presenta adecuados índices de validez (Calero, 2013).

Cuestionario Sociodemográfico (ad hoc). Diseñado para caracterizar a la muestra en función de variables sociodemográficas (edad, sexo y características de las actividades extracurriculares en que participa). En función de los objetivos de este trabajo se consideraron sólo las actividades extracurriculares evaluadas, que eran autodeterminadas y se correspondian con actividades artísticas o actividades deportivas. Se evalúo el tiempo desde que comenzaron a practicar la actividad; el tiempo dedicado semanalmente a la actividad; si realizaban la actividad con amigos y si tenían un profesor que coordinaba la actividad. Las variables relacionadas con el tiempo fueron definidas en función de los resultados obtenidos en una muestra piloto de la misma población.

\section{Procedimiento}

La bateria de instrumentos autoinformados fue entregada personalmente a los adolescentes en un sobre cerrado. Ellos debian contestar en forma anónima. La administración se realizó durante el horario de clase dentro de la institución educativa, evitando la presencia de docentes que pudieran afectar la respuesta de los adolescentes.

Cada uno de los inventarios administrados contaba con las instrucciones necesarias para responder correctamente a cada una de las preguntas $o$ afirmaciones. 


\section{Análisis de datos}

La prueba de Kolmogorov-Smirnov indica que los valores de la distribución se alejan significativamente de los percentiles teóricos de una distribución normal asintótica (ver tabla 1). Por esta razón se decidió emplear estadísticos que tengan como referente parámetros no normales. Se aplicó una prueba $U$ Mann Whitney para evaluar las diferencias en el nivel de inteligencia emocional en función de la participación y características de las actividades extracurriculares. Para evaluar el efecto de la significación estadística de las diferencias obtenidas entre los grupos se utilizó el estadístico Delta de Cliff, el cual permite cuantificar la magnitud de la diferencia entre dos variables cuando estas no cumplen con el supuesto de normalidad. Este estadístico da un índice entre $1 \mathrm{y}-1$, cuanto más se acerca a estos extremos más importante es el tamaño del efecto (Cliff, 1993; Macbeth, Razumiejczyk, \& Ledesma, 2010).

Tabla 1

Descriptivos. Prueba de normalidad Kolmogorv-Smirnov.

\begin{tabular}{lccccccc}
\hline & $M$ & $D E$ & Curtosis & Asimetría & K-S & gl & $p$ \\
\cline { 2 - 7 } & 3.64 & 0.67 & 0.12 & -0.35 & 0.07 & 398 & $<.001$ \\
Atención a los sentimientos & 3.39 & 0.74 & -0.06 & -0.47 & 0.10 & 398 & $<.001$ \\
Repridad emocional & 3.57 & 3.57 & -0.11 & -0.45 & 0.09 & 398 & $<.001$ \\
\hline
\end{tabular}

\section{RESULTADOS}

Se encontraron diferencias significativas entre los adolescentes que realizaban y no realizaban actividades extracurriculares en el componente de inteligencia emocional claridad $(U=12634.000 ; Z=-2.23 ; p=.03)$ (ver tabla 2). La diferencia en el componente claridad se da por un mayor índice en quienes participan en actividades extracurriculares. El estadístico delta de Cliff muestra un tamaño del efecto bajo para la diferencia entre los que participan y no participan en actividades extracurriculares en el factor claridad (-.15).

Tabla 2

IE percibida según la participación en actividades extracurriculares.

\begin{tabular}{lccccc}
\hline & \multicolumn{3}{c}{ Rango Promedio } & & \\
\cline { 2 - 5 } & $\begin{array}{c}\text { Participan } \\
(n=300)\end{array}$ & $\begin{array}{c}\text { No participan } \\
(n=99)\end{array}$ & $U$ & $Z$ & $p$ \\
\hline Atención & 205.37 & 183.73 & 13239.50 & -1.62 & .11 \\
Claridad & 207.39 & 177.62 & 12634.00 & -2.23 & .03 \\
Reparación & 199.81 & 200.59 & 14792.00 & -0.06 & .95 \\
\hline Nota: $U=U$ de Mann Whitney & & & & &
\end{tabular}

Se analizó si la inteligencia emocional mostraba características diferenciales en función de que las actividades sean artísticas o deportivas. Se encontró que en claridad existían diferencias significativas, siendo menor para quienes participaban en actividades artísticas $(U=7887.50 ; Z=-3.23 ; p=.00)$ (ver tabla 3). El estadístico delta de Cliff muestra un tamaño del efecto medio 
para la diferencia entre los que participan en actividades artísticas y deportivas para el factor claridad (-.48).

Tabla 3

IE percibida según la actividad extracurricular sea deportiva o artística.

\begin{tabular}{ccccccc}
\hline & \multicolumn{2}{c}{ Rango Promedio } & & & \\
\cline { 2 - 5 } & $\begin{array}{c}\text { Actividad artística } \\
(n=116)\end{array}$ & $\begin{array}{c}\text { Actividad } \\
\text { deportiva } \\
(n=175)\end{array}$ & $U$ & $Z$ & $p$ \\
\hline Atención & 147.78 & 144.82 & 9943.50 & -0.30 & .77 \\
Claridad & 126.50 & 158.93 & 7887.50 & -3.23 & .00 \\
Reparación & 143.18 & 147.87 & 9823.00 & -0.47 & .64 \\
\hline
\end{tabular}

Nota: $U=U$ de Mann Whitney

No se hallaron diferencias significativas al estudiar si existian diferencias en los niveles de inteligencia emocional en función de si practicaban la actividad desde hacía menos o más de dos años, como así tampoco por el tiempo semanal dedicado (hasta 3 o más horas; ver tabla 4).

\section{Tabla 4}

IE percibida y características relacionadas con el tiempo dedicado a la participación en actividades extracurriculares.

\begin{tabular}{|c|c|c|c|c|c|}
\hline & \multicolumn{2}{|c|}{ Rango Promedio } & \multirow[b]{3}{*}{$U$} & \multirow[b]{3}{*}{$Z$} & \multirow[b]{3}{*}{$p$} \\
\hline & \multicolumn{2}{|c|}{ La realiza desde... } & & & \\
\hline & $\begin{array}{l}\text { Hasta } 2 \text { años } \\
\quad(n=188)\end{array}$ & $\begin{array}{l}\text { Más de } 2 \text { años } \\
\quad(n=110)\end{array}$ & & & \\
\hline Atención & 151.79 & 148.16 & 10088.50 & -0.35 & .73 \\
\hline Claridad & 148.87 & 149.87 & 10270.50 & -0.10 & .92 \\
\hline \multirow[t]{3}{*}{ Reparación } & 138.29 & 156.06 & 9106.50 & -1.72 & .09 \\
\hline & \multicolumn{2}{|c|}{ Dedica semanalmente... } & & & \\
\hline & $\begin{array}{l}\text { Hasta } 3 \text { horas } \\
\quad(n=159)\end{array}$ & $\begin{array}{l}\text { Más de } 3 \text { horas } \\
\quad(n=138)\end{array}$ & $U$ & $Z$ & $p$ \\
\hline Atención & 151.31 & 146.34 & 10603.50 & -0.50 & .62 \\
\hline Claridad & 145.76 & 152.73 & 10456.50 & -0.70 & .49 \\
\hline Reparación & 157.06 & 139.71 & 9689.000 & -1.74 & .08 \\
\hline
\end{tabular}

El hecho de que la actividad esté dictada por un profesor o el realizar la actividad con amigos tampoco afectó los niveles de inteligencia emocional. Solo se observó una diferencia marginal en atención $(U=3652.500 ; Z=-1.868 ; p=$ .062) siendo mayor en aquellos adolescentes que tenían profesor (ver tabla 5). 
Tabla 5

IE percibida según características relacionadas con los aspectos sociales de la participación en actividades extracurriculares.

\begin{tabular}{|c|c|c|c|c|c|}
\hline & \multicolumn{2}{|c|}{ Rango Promedio } & \multirow[b]{2}{*}{$U$} & \multirow[b]{2}{*}{$Z$} & \multirow[b]{2}{*}{$p$} \\
\hline & $\begin{array}{c}\mathbf{S i} \\
(n=213)\end{array}$ & $\begin{array}{c}\text { No } \\
(n=81) \\
\end{array}$ & & & \\
\hline Atención & 147.02 & 148.76 & 8524.50 & -.16 & .88 \\
\hline Claridad & 144.20 & 156.17 & 7924.00 & -1.08 & .28 \\
\hline \multirow[t]{3}{*}{ Reparación } & 148.49 & 144.91 & 8416.50 & -.32 & .75 \\
\hline & \multicolumn{2}{|c|}{ Tiene profesor... } & & & \\
\hline & $\begin{array}{c}\mathbf{S i} \\
(n=259)\end{array}$ & $\begin{array}{c}\text { No } \\
(n=35)\end{array}$ & $U$ & $Z$ & $p$ \\
\hline Atención & 150.90 & 122.36 & 3652.50 & -1.87 & .06 \\
\hline Claridad & 145.57 & 161.80 & 4032.00 & -1.06 & .29 \\
\hline Reparación & 148.17 & 142.53 & 4358.50 & -.37 & .71 \\
\hline
\end{tabular}

\section{DISCUSIÓN}

La inteligencia emocional, desde el modelo de Salovey et al. (1995), se define como una serie de habilidades en el manejo de las emociones que permiten al sujeto servirse de la información que estas brindan y lograr una mejor adaptación al contexto. La inteligencia emocional percibida está compuesta por: la percepción del sujeto sobre la atención que presta a sus emociones, la percepción sobre la claridad con que percibe las emociones y la percepción sobre la propia capacidad de reparar los estados de ánimo negativos. Desde este punto de vista, las habilidades que componen la inteligencia emocional son aprendidas en la interacción con el entorno. Asimismo, durante la adolescencia la existencia de contextos seguros y estables emocionalmente se ha visto asociada a un mejor manejo de las emociones (Larson \& Brown, 2007). Por esta razón, el objetivo de este trabajo fue comparar la inteligencia emocional percibida según se participe o no en actividades extracurriculares deportivas o artísticas. Con este fin se analizó si la participación o las características de las actividades extracurriculares se relaciona con los niveles de inteligencia emocional percibida.

En relación con el primer objetivo general, se encontró en la muestra total que los adolescentes que realizaban actividades extracurriculares reportaron percibirse con mayor claridad a la hora de comprender sus emociones. Lo cual puede deberse a que el aprendizaje que los adolescentes realizan en estas actividades los ayudaria a desarrollar herramientas para poder desglosar y etiquetar con mayor claridad sus emociones.

Las investigaciones previas, que analizaron lo planteado en el segundo objetivo específico en relación con variables distintas de la inteligencia emocional, habían hallado que aquellos adolescentes que participaban en varias actividades extracurriculares de forma simultánea tenían un mayor ajuste por el desarrollo de competencias fisicas, psicológicas, sociales y emocionales que serían aplicables a distintas áreas personales y, también, porque de esta manera podían compensar experiencias negativas vivenciadas en una actividad en particular (Fredricks \& Eccles, 2006). Por ejemplo, sería 
esperable que aquellos adolescentes que practican más de una actividad tengan mejores niveles de autoestima al ingresar a la adultez dado que las habilidades desarrolladas y los autoconceptos asociados en cada actividad tienen más facilidad para ser transferidos a la adultez que aquellos que solo practican una actividad (Kort-Butler \& Hagewen, 2011). En el presente estudio, en función del segundo objetivo específico propuesto, a diferencia de lo planteado por Fredricks y Eccles (2006), no se hallaron diferencias entre los adolescentes que participaban en una o más actividades en los niveles de inteligencia emocional percibida. Es decir, que el tener más de un contexto en el que realizar aprendizajes y compensar experiencias negativas no parece afectar los niveles de inteligencia emocional percibida.

Tampoco se hallaron diferencias en lo planteado por el tercer objetivo específico, los niveles de inteligencia emocional percibida no se diferenciaron significativamente ni en función del tiempo desde que hacían la actividad ni por el tiempo dedicado a la misma. Adachi y Willoughby (2014), refiriéndose a la relación de la participación en actividades extracurriculares con la autoestima, habian restado importancia a la frecuencia de la práctica primando el disfrute que los adolescentes experimentan en la realización de estas actividades. Parece que aquello que influye en los niveles de claridad que los adolescentes reportaron no recae sobre variables relacionadas con el tiempo dedicado a estas actividades, de forma similar a lo que plateaban Adachi y Willoughby (2014) en relación a la autoestima.

Asimismo, en relación con el cuarto objetivo específico propuesto, se encontró que los adolescentes que participaban en actividades artísticas reportaron menores índices de claridad. Si se examinan las características de las actividades deportivas, estas suelen tener un perfil más dirigido a un objetivo, mientras que en las artísticas se suele aceptar y, hasta promover, un mayor nivel de introspección. Podría caber el interrogante sobre si esta diferencia en la claridad emocional a favor de los que realizan actividades extracurriculares deportivas no se debe a que los que realizan actividades artísticas son más críticos a la hora de juzgar su comprensión de las emociones. En estas actividades, por las características propias que posee, se espera que los adolescentes estén más atentos a sus emociones, perciban más emociones y, quizás por esta razón, perciban que tienen un menor nivel de claridad para etiquetar sus emociones que aquellos adolescentes que realizan actividades deportivas.

Cuando se estudió si el hecho de que la actividad fuese dictada por un profesor afectaba los niveles de inteligencia emocional percibida, tal como se propuso en el quinto objetivo específico, solo en el caso del componente atención se observó una tendencia que indicaba que era mayor para aquellos adolescentes que tenían profesor. Como se trata solo de una tendencia, la interpretación se debe realizar con cautela, pero quizás un profesor que guíe la actividad pueda enseñar a los adolescentes a atender a sus emociones para poder utilizar la información que éstas brindan para guiar sus comportamientos.

En la actualidad se le brinda una mayor importancia a las actividades y contextos adolescentes, por el papel fundamental que los mismos desempeñan en el desarrollo (Benson, 2003; Coleman \& Hendry, 2003; Hansen et al. 2003). Durante las últimas décadas, el estudio de la adolescencia viró hacia un enfoque basado en el desarrollo positivo, en el que se destaca el desarrollo de competencias, en la interacción con el contexto (Lerner, 2002; Thoekas et al., 
2005). Por otro lado, las habilidades emocionales que el adolescente desarrolle en la interacción con el medio y que posea ejercerán influencia sobre la autorregulación funcional necesaria para administrar y utilizar de forma adecuada la información emocional para lograr una mayor adaptación y bienestar con el contexto. Por ende, el estudio de aquellos factores que pueden potenciar un desarrollo de las habilidades emocionales adecuado se vuelve prioritario tomando en consideración las problemáticas adolescentes actuales, desde el consumo de alcohol hasta conductas autolescivas.

El hecho de que los adolescentes que participen en actividades extracurriculares reporten una percepción de mayor capacidad para entender sus emociones puede facilitar su comprensión de las emociones en otras personas. Mayer, DiPaolo y Salovey (1990) observaron que la posibilidad de identificar las emociones en otras personas posibilita entender sus estados emocionales, mostrando ser un constructo muy importante en la existencia de la empatia. De hecho, se considera que las personas emocionalmente inteligentes son más hábiles para percibir, comprender y manejar sus propias emociones, lo cual deriva en relaciones interpersonales más sanas debido a que extrapolan sus habilidades de comprensión a las emociones de los demás (Extremera Pacheco \& Fernández-Berrocal, 2004). Por ende, los resultados obtenidos en esta investigación refuerzan la importancia de las actividades extracurriculares en el desarrollo de habilidades en el adolescente que favorecen un crecimiento saludable como individuos sociales.

\section{CONCLUSIÓN}

Este trabajo representa una primera aproximación sobre un tópico joven, como la inteligencia emocional, y un área de estudio como la adolescencia con un enfoque centrado en el desarrollo positivo. Desde esta perspectiva, el análisis de los contextos que los adolescentes transitan y su contribución al desarrollo permite conocer aquellos factores que se pueden potenciar desde posibles intervenciones dirigidas a tal fin.

Como posibles líneas de investigación que surgen del presente trabajo se plantea la necesidad de replicarlo tomando en consideración otro tipo de actividades, como las comunitarias o religiosas, por ejemplo. Asimismo, estudiar la relación entre la inteligencia emocional y la participación en actividades en distintos extractos socioeconómicos resultaría interesante dada la importancia que las habilidades emocionales poseen para el ajuste psicológico.

Esta investigación no se encuentra exenta de limitaciones: la muestra corresponde a una población de adolescentes de la Ciudad Autónoma de Buenos Aires, por lo que es necesario ampliar la población para poder aumentar la validez externa de investigación. También, existen limitaciones, ya que no fueron evaluadas características que las actividades podrian tener como diferencias cualitativas en la realización profesional o recreativa o en relación con el nivel de estructura de las actividades.

\section{REFERENCIAS}

Adachi, P. J. \& Willoughby, T. (2014). It's not how much you play, but how much you enjoy the game: The longitudinal associations between adolescents' self-esteem and the frequency versus enjoyment of involvement in sports. Journal of youth and adolescence, 43(1), 137-145. doi: 10.1007/s10964-0139988-3

Benson, P. L. (2003). Developmental assets and asset-building community: Conceptual and empirical 
foundations. En R. M. Lerner \& P. L. Benson (Eds.), Developmental assets and asset-building communities (pp. 19-43). Boston: Springer. https://doi.org/10.1007/978-1-4615-0091-9_2

Calero, A. D. (2013). Versión Argentina de la Trait Meta Mood Scale (TMMS) para adolescentes: Una medida de la inteligencia emocional percibida. Cuadernos de Neuropsicologia- Panamerican Journal of Neuropshychology, 7(1), 104-119. . https://doi.org/10.7714/cnps/7.1.206

Calero, A. D. (2016). Actividades extraescolares durante la adolescencia: características que facilitan las experiencias óptimas. Psicoperspectivas, 15(2), 102-109. https://doi.org/10.5027/psicoperspectivasVol15-Issue2-fulltext-856

Cliff, N. (1993). Dominance statistics: Ordinal analyses to answer ordinal questions. Psychological Bulletin, 114, 494-509. https://doi.org/10.1037/0033-2909.114.3.494

Coleman, J. C. \& Hendry, L. B. (2003). Psicologia de la adolescencia. Madrid: Ediciones Morata.

Damasio, A. R. (1994). El error de Descartes. Barcelona: Crítica.

Eccles, J. S. \& Gootman, J. A. (2002). Community programs to promote youth development. Washington DC: National Academy Press.

Extremera Pacheco, N. \& Fernández-Berrocal, P. (2004). El papel de la inteligencia emocional en el alumnado: evidencias empíricas. Revista Electrónica de Investigación Educativa, 6(2), 1-17.

Fernández-Berrocal, P., Salovey, P., Vera, A., Ramos Díaz, N., \& Extremera Pacheco, N. (2002). Cultura, inteligencia emocional percibida y ajuste emocional: un estudio preliminar. Revista Española de Motivación y Emoción, 3, 159-167.

Fredricks, J. A., \& Eccles, J. S. (2006). Is extracurricular participation associated with beneficial outcomes? Concurrent and longitudinal relations. Developmental psychology, 42(4), 698. https://doi.org/10.1037/0012-1649.42.4.698

Gracia, E., \& Herrero, J. (2006). La comunidad como fuente de apoyo social: evaluación e implicaciones en los ámbitos individual y comunitario. Revista Latinoamericana de Psicología, 38(2), 327-342.

Goleman, D. (2012). Inteligencia emocional. Barcelona: Editorial Kairós.

Hansen, D. M., Larson, R. W., \& Dworkin, J. B. (2003). What adolecents learn in organized youth activities: A survey of Self-Reported developmental experiences. Journal of Research on Adolescence, 13(1), 25-55. https://doi.org/10.1111/1532-7795.1301006

Hernández Sampieri, R., Fernández-Collado, C., \& Baptista Lucio, P. (2008). Metodología de la investigación. (4ta ed.). México: Mc Graw Hill.

Jiménez, M. I. \& López-Zafra, E. (2011). Actitudes sociales y adaptación social en adolescentes españoles: el papel de la inteligencia emocional percibida. Revista de Psicología Social, 26(1), 105-117. https://doi.org/10.1174/021347411794078417

Kort-Butler, L. A. \& Hagewen, K. J. (2011). School-based extracurricular activity involvement and adolescent self-esteem: A growth-curve analysis. Journal of youth and adolescence, 40(5), 568-581. https://doi.org/10.1007/s10964-010-9551-4

Larson, R. W. (2000). Toward a Psychology of Positive youth Development. American Psychologist, 55, 170183. https://doi.org/10.1037/0003-066X.55.1.170

Larson, R. W. \& Brown, J. R. (2007). Emotional development in adolescence: What can be learned from a high school theater program? Child Development, 78(4), 1083-1099. https://doi.org/10.1111/j.14678624.2007.01054.x

Lerner, R. M. (2002). Concepts and theories of human development Lawrence Erlbaum Associates ( $3^{\mathrm{a}}$ ed ed.). Mahwah, NJ: Psychology Press.

Lozano, A. B., Uzquiano, M. P., Rioboo, A. M. P., Paz, R. S., \& Castro, F. V. (2013). Estrategias de aprendizaje, autoconcepto y rendimiento académico en la adolescencia. Revista galego-portuguesa de psicoloxía e educación: revista de estudios e investigación en psicología y educación, 21, 195-212.

Macbeth, G., Razumiejczyk, E., \& Ledesma, R. D. (2010). Cliff s Delta Calculator: Un programa para calcular el tamaño del efecto no paramétrico al comparar dos grupos de observaciones. Universitas Psychologica, 10(2), 545-555.

Mayer, J. D., DiPaolo, M., \& Salovey, P. (1990). Perceiving affective content in ambiguous visual stimuli: A component of emotional intelligence. Journal of personality assessment, 54(3-4), 772-781. doi: $10.1207 / \mathrm{s} 15327752 \mathrm{jpa} 5403 \& 4 \_29$

Mayer, J. D. \& Salovey, P. (1997). What is emotional intelligence? En P. Salovey \& D. J. Sluyter (Eds.), Emotional development and emotional intelligence: Implications for educators (pp. 3-31). New York: Basic Books.

Mayer, J. D., Salovey, P., \& Caruso, D. (2000). Models of Emotional intelligence. En I. R. Sternberg (Ed.), The handbook of intelligence (pp. 396-422). Cambridge: Cambridge University Press. https://doi.org/10.1017/CBO9780511807947.019

Palomera, R., Salguero, J. M. \& Fernández-Berrocal, P. (2011). ¿Es importante la inteligencia emocional percibida para a adaptación y bienestar infanto-juvenil? En P. Fernández-Berrocal, N. Extremera, R. Palomera, D. Ruíz-Aranda, J. M. Salguero \& R. Cabello (Eds.), Inteligencia Emocional: 20 años de investigación y desarrollo. Santander: Fundación Marcelino Botín.

Parra Jiménez, A., Oliva Delgado, A., \& Antolín Suárez, L. (2009). Los programas extraescolares como recurso para formentar el desarrollo positivo adolescente. Papeles del Psicólogo, 30(3), 3-13.

Rey, L., Extremera Pacheco, N., \& Pena, M. (2011). Perceived emotional intelligence, self-esteem and life satisfaction in adolescents. Psychosocial Intervention, 20(2), 227-234. https://doi.org/10.5093/in2011v20n2a10

Salguero, J. M., Fernández-Berrocal, P., Ruiz-Aranda, D., Castillo, R., \& Palomera, R. (2011). Inteligencia emocional y ajuste psicosocial en la adolescencia: El papel de la percepción emocional. European Journal of Education and Psychology, 4(2), 143-152.

Salguero, J. M., Palomera, R., \& Fernández-Berrocal, P. (2012). Perceived emotional intelligence as predictor 
of psychological adjustment in adolescents: a 1-year prospective study. European Journal of Psychology of Education, 27(1), 21-34. https://doi.org/10.1007/s10212-011-0063-8

Salovey, P. \& Mayer, J. D. (1990). Emotional intelligence. Imagination, Cognition, and Personality, 9, 185211. https://doi.org/10.2190/DUGG-P24E-52WK-6CDG

Salovey, P., Mayer, J. D., Goldman, S. L., Turvey, C., \& Palfai, T. P. (1995). Emotional attention, clarity, and repair: Exploring emotional intelligence using the trait meta-mood scale. En J. W. Pennebaker (Ed.), Emotion, disclosure \& health (pp. 125-151). Washington: American Psychological Association. https://doi.org/10.1037/10182-006

Thoekas, C., Almerigi, J., Lerner, R. M., Dowling, E. M., Benson, P. L., Scales, P. C., \& Von Eye, A. (2005). Conceptualizing and modeling individual and ecological asset components of thriving in early adolescence. Journal of Early Adolescence, 25(113-143). https://doi.org/10.1177/0272431604272460

Williams, F. M., Fernández-Berrocal, P., Extremera Pacheco, N., Ramos Díaz, N., \& Joiner Jr, T. E. (2004). Mood regulation skill and the symptoms of endogenous and hopelessness depression in Spanish high school students. Journal of Psychopathology and Behavioral Assessment, 26(4), 233-240. https://doi.org/10.1023/B:JOBA.0000045339.67766.86

Recibido 30-05-2017 | Aceptado 10-08-2017 
Calero, A. D., Barreyro, J. P., \& Injoque-Ricle, I. / Psicodebate, 17(2), 43 - 54.

Psicodebate, Vol. 17, No 2, Diciembre 2017 - Mayo 2018

ISSN: 1515-2251 e-ISSN: 2451-6600 